and one case of synchronous regional and distant failure were identified. The crude failure rate was $7.1 \%$, which is higher than rates reported in previous series; however, two of the patients who experienced failure would not have been eligible for PBI in accordance with American Brachytherapy Society treatment recommendations. Exclusion of these patients from the analysis yields a failure rate of $4.4 \%$, which is more consistent with earlier reports.

The authors conclude that the careful selection of patients for $\mathrm{PBI}$ is of paramount importance for achieving good outcomes. The results of the NSABP B-39/RTOG 0413 trial should help to further define the subgroup of patients for whom MammoSite ${ }^{\circledR}$ PBI would be most beneficial.

Original article Chen S et al. (2007) Patterns of failure after MammoSite brachytherapy partial breast irradiation: a detailed analysis. Int J Radiat Oncol Biol Phys 69: 25-31

\section{High diagnostic sensitivity of MRI for pure DCIS}

MRI has traditionally been regarded as an inferior technique for the detection of ductal carcinoma in situ (DCIS) because of its failure to detect all cancers that are apparent on mammography. Recently, however, it has been reported that MRI might enable the prospective diagnosis of DCIS that is undetectable on mammography. Now, Kuhl et al. have investigated the relative diagnostic sensitivities of MRI and mammography for DCIS, and suggest that the former technique better identifies high-grade DCIS, which is more likely than low-grade DCIS to progress to high-grade invasive breast cancer.

In total, 7,319 women underwent breast MRI and mammography at a German tertiary referral center during the 5-year study period. A positive imaging result was found in 1,208 cases, with final surgical diagnoses of invasive breast cancer and pure DCIS made in 469 and 167 women, respectively. Significantly more cases of confirmed DCIS were detected by MRI than on mammography ( $92 \%$ vs $56 \% ; P<0.0001)$. DCIS was detected by both imaging techniques in $81(49 \%)$ cases, by mammography only in 12 (7\%) cases, and by MRI only in 72 (43\%) cases. Notably, MRI demonstrated high sensitivity for high-grade DCIS, detecting $98 \%$ of these lesions. By contrast, only $52 \%$ of high-grade DCIS was identified on mammography.
The authors conclude that mammography might often fail to detect pure DCIS, especially high-grade cancers. MRI might be useful in the diagnosis of these lesions, which are likely to progress to invasive breast cancer.

Original article Kuhl CK et al. (2007) MRI for diagnosis of pure ductal carcinoma in situ: a prospective observational study. Lancet 370: 485-492

\section{Low plasma IGFBP-1 levels correlate with increased risk of pancreatic cancer}

Insulin-like growth factor binding protein-1 (IGFBP-1) is an inhibitor of insulin-like growth factor-I (IGF-I). IGF-I is highly expressed in pancreatic cancer cell lines; however, previous studies have failed to show an association of this protein with pancreatic cancer. Low plasma levels of IGFBP-1 have been associated with obesity and a sedentary lifestyle, both factors that have been implicated in the development of pancreatic cancer. Wolpin et al. examined the utility of IGFBP-1 as a prognostic factor in pancreatic cancer.

The study included 144 patients with pancreatic cancer and 429 controls matched for age, sex, and smoking and fasting status, who participated in four US prospective cohort studies. After adjusting for risk factors such as plasma IGF-I, IGF-binding protein-3 and C-peptide, subjects with circulating IGFBP-1 levels in the lowest quartile had a relative risk (RR) of pancreatic cancer of 2.07 compared with those in the three highest quartiles. Individuals in the lowest IGFBP-1 quartile who were diagnosed $\geq 8$ years after collection of blood samples had an adjusted RR of 3.47 compared with individuals in the three highest quartiles. The influence of low IGFBP-1 was shown to be greater among never smokers (RR 3.30), participants with nonfasting plasma samples ( $\leq 8$ hours of fasting; RR 2.69), and among individuals with elevated plasma C-peptide (RR 2.32); however, none of these interactions was statistically significant.

The authors conclude that low circulating levels of IGFBP-1 independently predict the risk of pancreatic cancer, and call for further studies to examine the role of insulin and the IGF axis in this malignancy.

Original article Wolpin BM et al. (2007) Circulating insulinlike growth factor binding protein-1 and the risk of pancreatic cancer. Cancer Res 67: 7923-7928 